\title{
Infestación, daño y fluctuación poblacional de Phyllocnistis citrella Stainton (Lepidoptera: Gracillaridae) en Bahía Concha, Santa Marta (Colombia)
}

\author{
Infestation, damage, and population dynamics of \\ Phyllocnistis citrella Stainton (Lepidoptera: Gracillaridae) in \\ Bahia Concha, Santa Marta (Colombia)
}
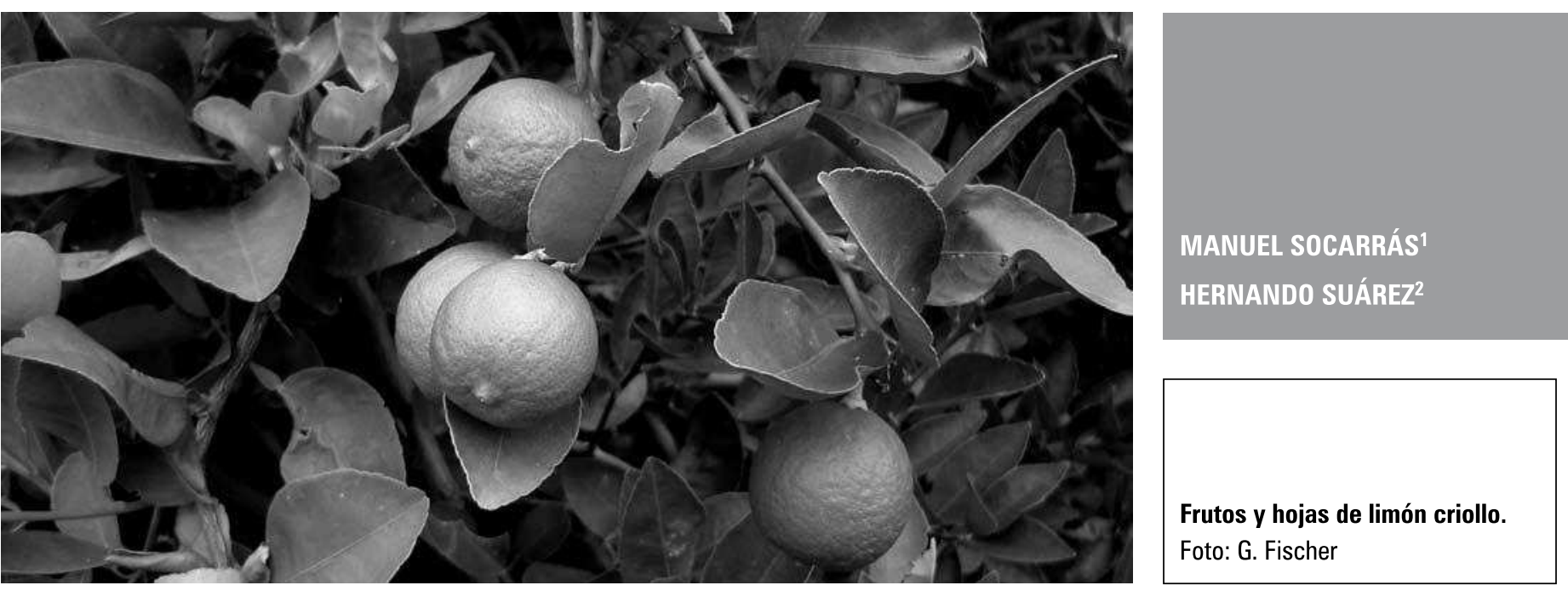

\section{RESUMEN}

La presente investigación se desarrolló en fases de campo y laboratorio, en la finca "El Centro" localizada en Bahía Concha, estribaciones del parque Tayrona de la Sierra Nevada de Santa Marta y en el laboratorio de entomología de la Universidad del Magdalena respectivamente durante el segundo semestre de 2004 y el primero de 2005. Durante ocho meses se determinó el porcentaje del daño, la infestación y la fluctuación poblacional, y se hicieron observaciones sobre enemigos naturales del minador de los cítricos Phyllocnistis citrella (Lepidoptera: Gracillaridae) en tres especies de cítricos: tangelo Minneola, mandarina Clementina y limón criollo sembrados en un lote de 14 ha. Los resultados mostraron un daño promedio de 30,95\%, con mayores lesiones en limón con $47 \%$ en el estrato medio de los árboles. En mandarina el daño fue de 36\% y en tangelo de $18 \%$ también en el estrato medio. Hubo diferencias significativas en el daño presentado en los estratos medio y alto, siendo mayor en limón y menor en tangelo. Los mayores picos poblacionales se presentaron en septiembre en mandarina y en noviembre en limón. Se encontró una relación directa entre precipitación y fluctuación poblacional, a mayor precipitación mayor se hace la población. En el laboratorio emergió un parasitoide identificado como Elasmus

Ingeniero Agrónomo, Programa de Ingeniería Agronómica, Universidad del Magdalena, Santa Marta (Colombia).

Profesor, Programa de Ingeniería Agronómica, Universidad del Magdalena, Santa Marta. hersugo@hotmail.com 
sp (Hymenoptera: Eulophidae). Así mismo a nivel de campo se encontró que los predadores más importantes fueron la avispa Polybia occidentalis (Hymenoptera: Vespidae) y el Chrysoperla sp. (Neuroptera: Chrysopidae).

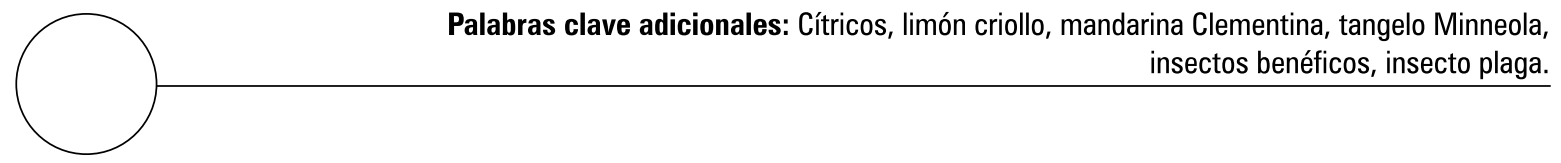

\section{ABSTRACT}

The research was conducted in the field and under laboratory conditions, in the farm El Centro located in Bahia Concha near the Tayrona park, Santa Marta, Colombia and laboratory of entomology of the University of Magdalena, respectively, during the first semester of 2004 and the second semester of 2005 . The damage percentage, infestation, population fluctuation were determined during eight months, as well as observations were made on the natural enemies of citrus crop miner Phyllocnistis citrella (Lepidoptera: Gracillaridae) in three species of citrus: tangelo Minneola, mandarin Clementina and common acid lime sown in the area of 14 ha. The results revealed average $30,95 \%$ damage with the highest damage in lime of $47 \%$ at the medium height of trees. In mandarin, the damage was $36 \%$ and $18 \%$ in tangelo at the medium height of trees. The main population peaks were the highest ones in September on mandarin and in November on lime. A direct relation between precipitation and population dynamics was observed. In laboratory, the parasitoid Elasmus sp. (Hymenoptera: Eulophidae) and, in field, the most important predator wasp Polybia occidentalis (Hymenoptera: Vespidae) were identified.

Additional key words: Citrus, common acid lime, mandarin Clementina, tangelo Minneola, beneficial insects, pest insect.

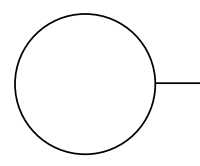

INTRODUCCIÓN

Los cítricos pertenecen al género Citrus y a la familia Rutaceae. Entre las especies más conocidas sobresalen: naranjas, limones, mandarinas y pomelos. El origen del género Citrus se sitúa en el sureste de Asia y el centro de China, Filipinas y el Archipiélago Indo-malayo hasta Nueva Guinea.

Los principales productores de cítricos en el mundo son Brasil y Estados Unidos con 22,6 y $15,6 \%$ respectivamente de la producción mundial; les siguen en importancia China, México, España e India. Estos seis países son responsables del $64,17 \%$ del total.

Colombia figura en el puesto 29 con una participación de $0,37 \%$ del total y una tasa de crecimiento anual de 5,06\%.

Los cítricos son cultivos de gran importancia a nivel mundial por su valor alimenticio, los productores utilizan la fruta para extracción y producción de jugos, algunos aceites, esencias uti- 
lizadas en perfumerías y comercialización de la fruta en sí.

La producción del cultivo de cítricos en Colombia y en el mundo enfrenta diversas dificultades, factores bióticos y abióticos, los cuales influyen en la productividad.

Organismos como los insectos, en diferentes poblaciones pueden ser perjudiciales o benéficos a los cultivos. Entre los insectos plagas que atacan los cítricos, merece especial atención el minador de las hojas, hoy en día encontrado en cualquier parte del mundo donde se cultiven estos frutales. El minador de los cítricos $P$. citrella es un microlepidóptero perteneciente a la familia Gracillaridae y a la subfamilia Phyllocnistinae, considerado en la actualidad como uno de los insectos plaga de mayor importancia y repercusión sobre los cítricos a nivel mundial, debido a la gravedad de los daños que sobre ellos origina (Hepper, 1993).

La dispersión del minador de los cítricos a nivel mundial, desde la zona de origen, fue lenta hasta 1993, a partir de ese año y en poco tiempo la distribución de la plaga sufrió grandes cambios, de tal forma que en la actualidad existen muy pocas regiones donde se cultiven cítricos en que la presencia de la plaga no haya sido constatada. En América fue descubierto por primera vez en 1993 en varios viveros del estado de Florida (Heppner y Dixon, 1995).

En marzo de 1995, se reportó por primera vez el minador de las hojas de los cítricos en la zona central cafetera colombiana (Castaño, 1996). Pocos insectos plaga han tenido una expansión tan grande y han creado tanto desconcierto en un periodo tan corto. En cítricos se han realizado grandes esfuerzos para el estudio de los insectos plaga que atacan estos frutales, debido principalmente a que son uno de los cultivos que más se comercializan en el país. Las escamas, áfidos, ácaros y desfoliadores han sido tratados por varios autores (Bustillo, 1970).
Durante los últimos 25 años, la dispersión del insecto minador de las hojas de los cítricos ha aumentado, pudiendo confirmarse en la actualidad su presencia sobre los cítricos en los cinco continentes (Espadas, 1995).

Debido al difícil control de las poblaciones de larvas del minador, por su localización dentro de las hojas, por la permanente presencia de brotes foliares y abuso de agroquímicos que eliminan enemigos naturales, se han encontrado infestaciones por el minador tan elevadas que pueden llegar a 90-95\% de hojas minadas especialmente en viveros y en plantaciones recién establecidas (García, 1995).

En China, se han encontrado reducciones de hasta $50 \%$ de la producción y disminución del peso del fruto de 120 a $70 \mathrm{~g}$, los mismos estudios demuestran que si el minador destruye 30\% del área foliar, la producción se reduce un año mas tarde de haber ocurrido la infestación (García, 1995). El mismo autor sostiene que el daño del minador es a nivel foliar, esta pérdida afecta la tasa fotosintética ocasionando una reducción del rendimiento reflejado en menor cantidad de frutos o reducción de su peso, y además, se disminuye el contenido de ácidos, azucares y la cantidad de jugo.

Las plantaciones jóvenes injertadas y huertos con riego localizado, presentan mayores ataques del minador, por disponer continuamente de brotaciones nuevas, por lo que las estrategias de manejo del insecto se deben seguir de manera muy cuidadosa (Garrido, 1995).

El mismo autor reporta que la presencia del minador de las hojas de los cítricos, puede provocar que también cambie la importancia relativa de otras plagas, dado que el minador ocupa un nicho ecológico que antes de su aparición ocupaban otros fitófagos como pulgones, ácaros o moscas blancas. Sin embargo, se ha podido constatar que varias especies de insectos pueden desarrollarse incluso en hojas atacadas por el minador, ya que 
en las hojas enrolladas, han encontrado un lugar ideal para desarrollarse y refugiarse.

La expansión y futuro de la agricultura en la región Caribe depende en gran parte del desarrollo de nuevas estrategias de producción. Por tanto, existe la necesidad de revisar los conceptos actuales y que obstaculizan el aumento de la producción de alimentos y el mejoramiento del nivel socio-económico de la población campesina. (Suarez y Marín, 2002).

En el departamento de Magdalena se siembran aproximadamente 3.838 ha de cítricos y existe poca información local relacionada con el minador; es decir sus niveles poblacionales, el daño que puede estar ocasionando, materiales vegetales preferidos, época de aparición, ciclo de vida y enemigos naturales. Por estas razones, se realizó el presente trabajo, con miras a obtener mayor información relacionada con el insecto y poder realizar planes de manejo que garanticen una buena producción y una alta rentabilidad del cultivo en la zona.

\section{MATERIALES Y MÉTODOS}

El trabajo se desarrolló a nivel de campo en la finca "El Centro", situada en el sector de Bahía Concha, Santa Marta/ Magdalena, localizada dentro de los límites del parque Nacional Tayrona en las estribaciones de la Sierra Nevada de Santa Marta, y a nivel del laboratorio de Entomología de la Universidad del Magdalena, durante ocho meses a partir de septiembre de 2002 hasta abril de 2003.

Geográficamente la zona esta localizada entre las siguientes coordenadas $74^{\circ} 07^{\prime}$ y $74^{\circ} 12^{\prime}$ de longitud Oeste en el meridiano de Greenwich y $11^{\circ} 11^{\prime}$ y $11^{\circ} 14^{\prime}$ de latitud Norte con respecto al Ecuador.

En la finca hay sembradas 14 ha de cítricos: cuatro de limón criollo (C. aurantifolia), cuatro de mandarina Clementina ( $C$. clementina) y seis de tangelo Minneola (C. paradisi $x$ C. reticulata), sobre ellas se realizó un reconocimiento inicial y se determinó la presencia del minador. Se tomaron muestras de hojas con minas y se llevaron al laboratorio de entomología para confirmar la identificación de la especie, según las indicaciones y descripciones previas realizadas por Peña y Duncan (1993) y Castaño (1996).

La evaluación de la infestación y el daño del insecto en cada variedad de cítricos, se realizó según los protocolos reportados por Castaño (1996). Para el presente estudio, se marcaron tres árboles por especie de cítricos, sobre ellos una vez por semana, se tomó un brote de la parte media y un brote de la parte superior y sobre ellos en cada hoja presente se contó el número de larvas, número de pupas, minas activas y minas no activas. Esta parte del trabajo se realizó en el laboratorio mediante un estereoscopio. Las hojas con presencia de larvas del minador se colocaron en cámaras de observación para permitir la emergencia de posibles parasitoides. En el campo se realizo observación sobre presencia de predadores relacionados con el minador.

Se realizó un análisis de varianza y una prueba de Tukey $(P<0,05)$ para determinar la preferencia del minador por una especie de cítrico en particular (limón, mandarina o tangelo) considerando la población encontrada o el número de minas por árbol. Cada árbol constituyó una unidad experimental. La fluctuación de la población del minador en los tres materiales de cítricos, se correlacionó con la precipitación presentada en la zona durante el desarrollo de las observaciones.

\section{RESULTADOS Y DISCUSIÓN}

La población del $P$. citrella en la localidad estudiada, mostró alta densidad durante los ocho meses de estudio. De un total de 2.303 hojas revisadas (figura 1), 713 presentaron lesiones de larvas del minador, lo que corresponde a 30,95\%, las mayores lesiones se dieron en limón con 47,0\% en el estrato medio de los árboles y $38,0 \%$ en el 
estrato alto. Mandarina ocupó el segundo lugar con 36,0 y $34 \%$ respectivamente en los estratos medio y alto y por último el tangelo con 18 y $13 \%$ respectivamente (figura 2). Un análisis de varianza, mostró que hubo diferencias significativas en el daño presentado en los estratos medio ( $\mathrm{F}=$ 23,6120; $\mathrm{Gl}=2,0 ; P<0,05)$ y altos $(\mathrm{F}=16,4696$ : $\mathrm{Gl}=2 ; P<0,05)$ de los cultivares estudiados, con valores mayores en limón que en tangelo.

Cobo y Tróchez (1996a; 1996b), al estudiar la fluctuación del $P$. citrella en el Valle del Cauca sobre tangelo mineola, tangelo orlando, lima tahití y naranja valle washington, encontraron un daño superior al $20 \%$ en evaluaciones iniciales. Castaño (1996) afirma que en Colombia se han observado mayores ataques en naranja, lima y tangelo y en menor grado mandarinas.

La figura 3 muestra las minas activas (presencia de larvas) y las no activas (daño viejo) en las hojas de las plantas estudiadas. El porcentaje promedio de los cultivares estuvo por encima del
$30 \%$, lo que evidencia el daño ocasionado por el insecto, siendo tangelo la especie más afectada, seguida por limón y mandarina, respectivamente (figura 4). Sin embargo, el análisis de varianza no mostró diferencia significativa para la variable minas activas en los estratos medios ( $\mathrm{F}=$ $0,6838) ; \mathrm{Gl}=2 ; P>0,05)$ y superior $(\mathrm{F}=1,6588$; $\mathrm{Gl}=2 ; P>0,05)$ respectivamente entre los cultivares estudiados; además, se evidenció que, el minador puede provocar daño indistintamente en tangelo, mandarina o limón.

En el estudio no se determinó en las minas activas, qué cantidad de larvas, alcanzaron el estado adulto, ni tampoco posible parasitismo. Es importante resaltar la presencia de ácaros (Acari: Tetranychidae) en las minas no activas, lo cual se sustenta en que una vez ha emergido el adulto del minador, otros artrópodos. entre ellos los ácaros, invaden el área afectada, para alimentarse. La figura 5 muestra la fluctuación poblacional del minador durante el periodo de estudio, observándose que los mayores picos poblacionales

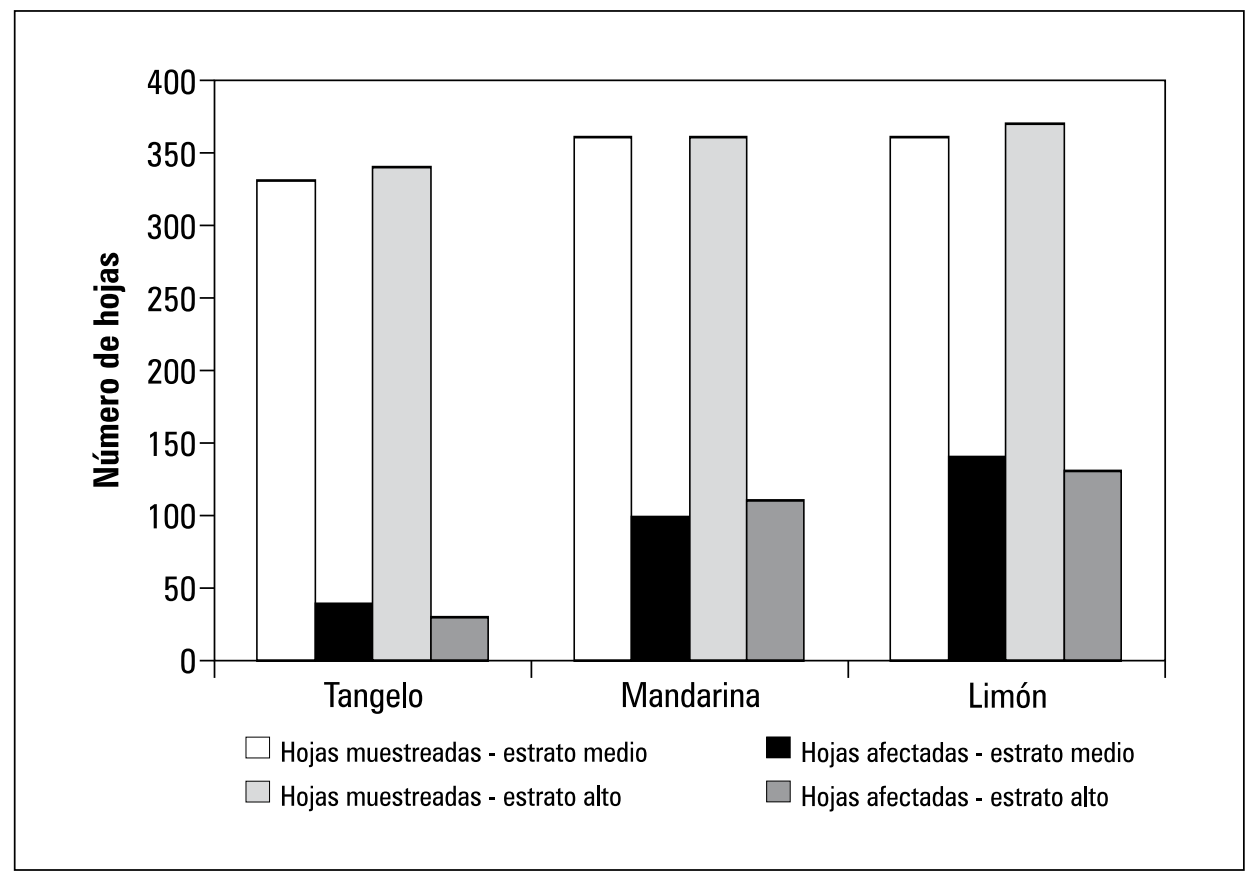

Figura 1. Número de hojas afectadas por $\boldsymbol{P}$. citrella en diferentes especies de cítricos. 


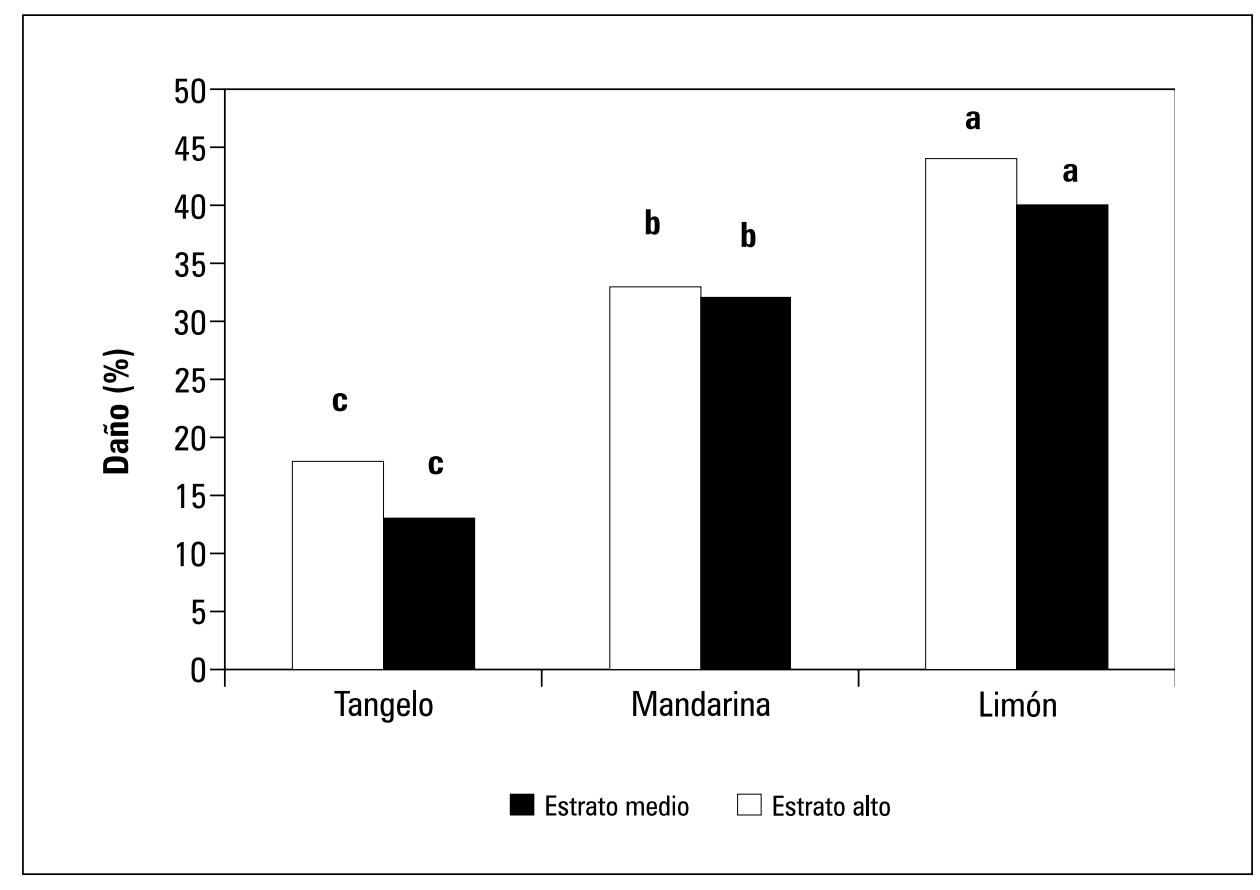

Figura 2. Porcentaje de daño en hojas causado por $\boldsymbol{P}$. citrella en diferentes especies de cítricos y porcentaje de daño (columnas de los estratos medios y altos de cada cultivar, respectivamente, seguidas por la misma letra no difieren significativamente, según prueba de Tukey $(P<0,05)$.

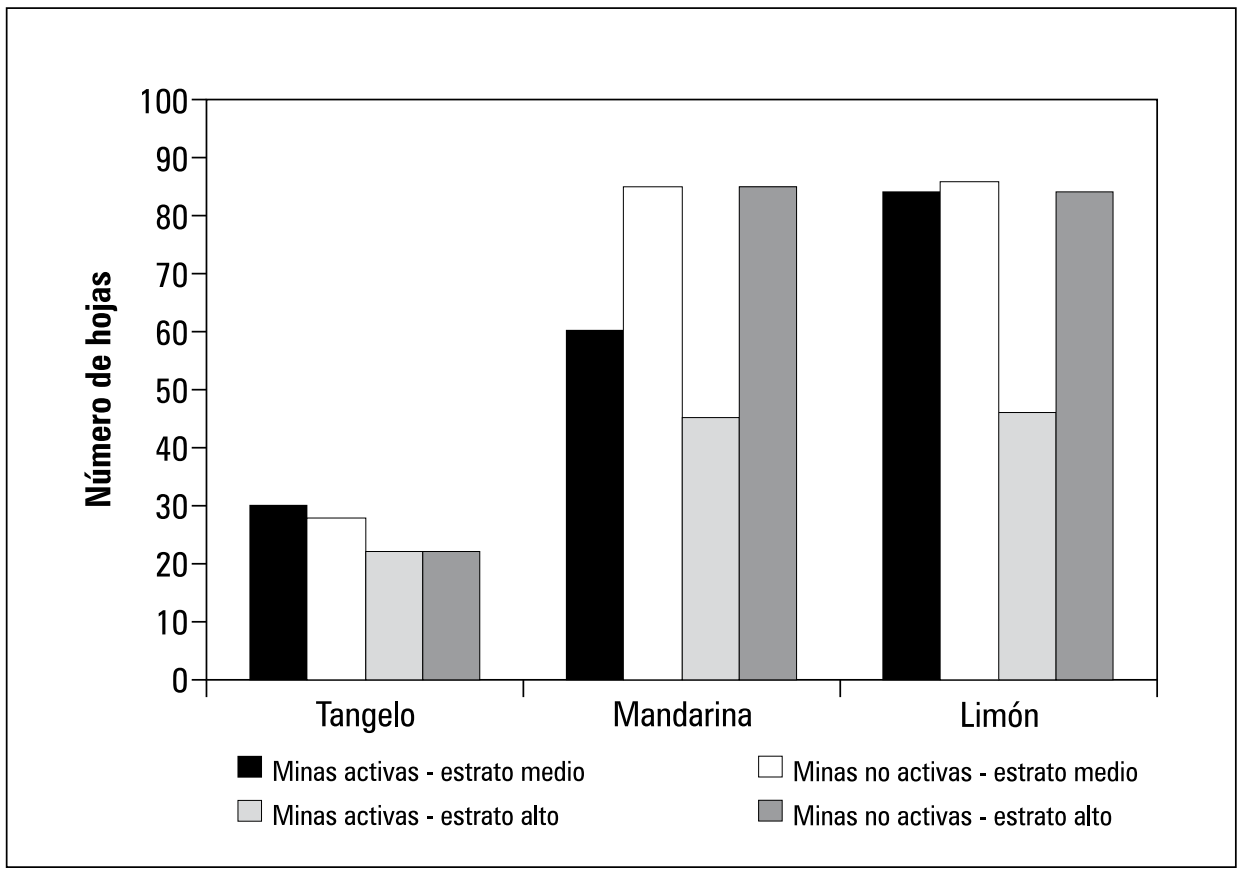

Figura 3. Minas activas causadas por $\boldsymbol{P}$. citrella los estratos medio y alto en diferentes especies de cítricos. 


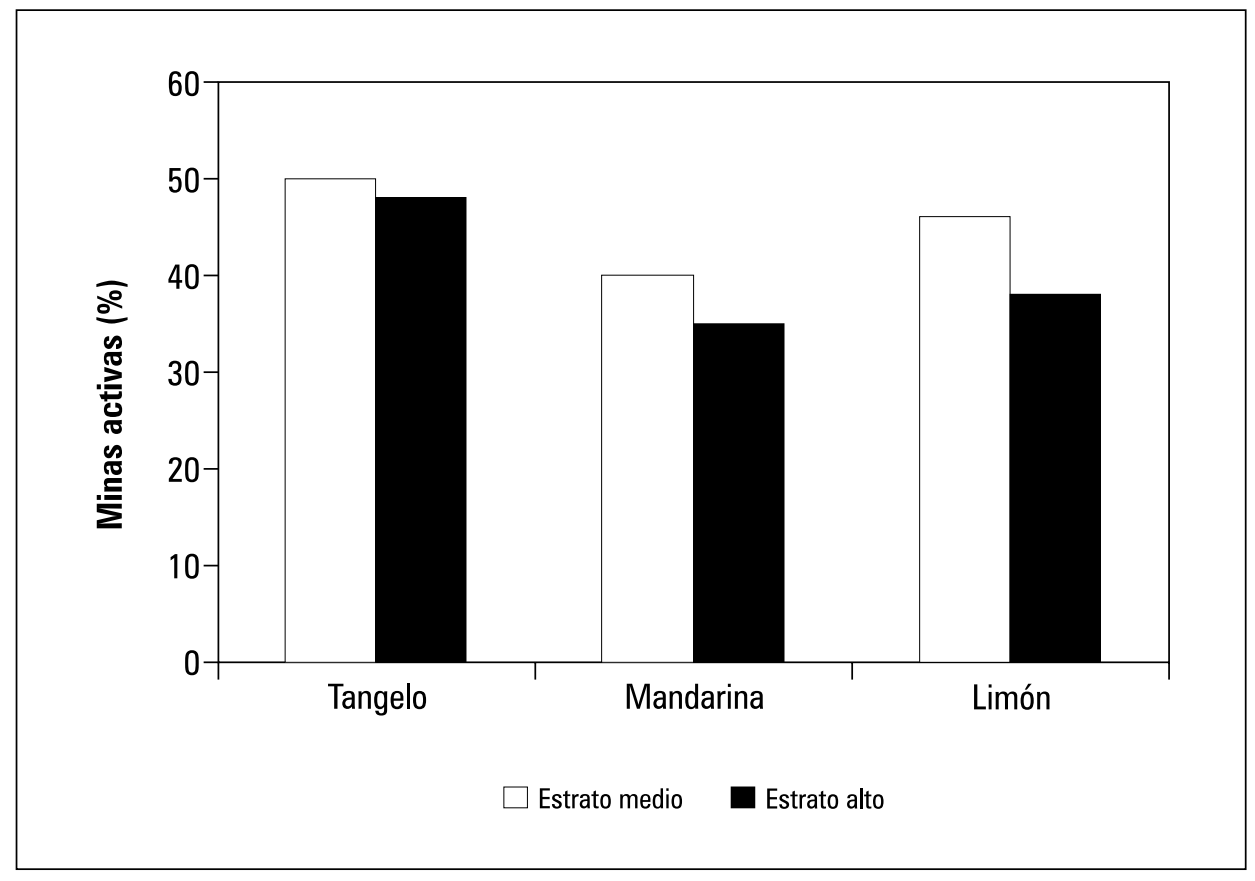

Figura 4. Porcentaje promedio de minas activas causadas por $\boldsymbol{P}$. citrella en los estratos medio y alto en diferentes especies de cítricos (para análisis estadístico los datos fueron transformados por arc seno $\sqrt{ } \mathbf{x})$.

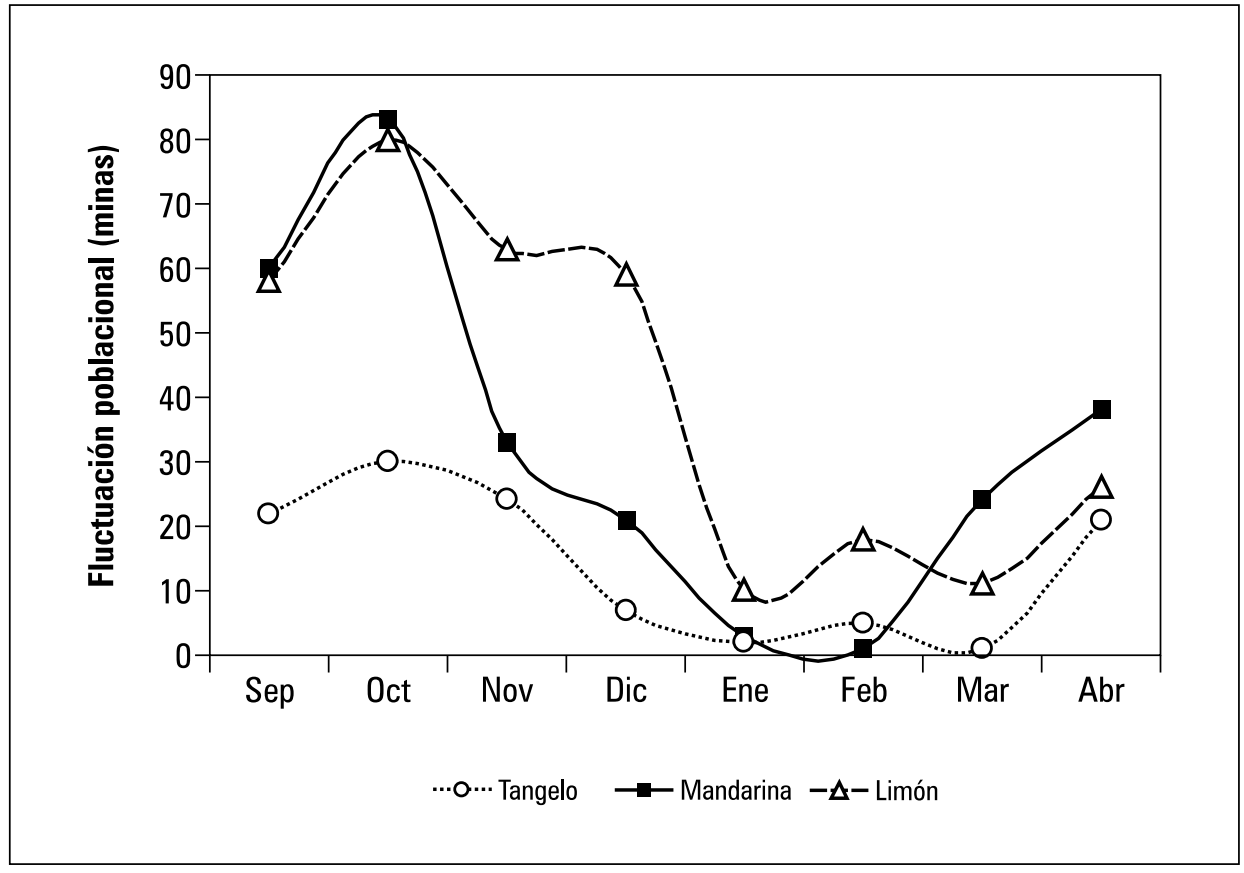

Figura 5. Fluctuación poblacional de $P$. citrella en diferentes especies de cítricos en Bahia Concha, municipio de Santa Marta, Magdalena, 2002-2003. 
se encontraron en septiembre y octubre en mandarina y en noviembre en limón. Posteriormente se presentó disminución de las poblaciones que comenzó en diciembre, se acentuó en enero, febrero y marzo de 2003 y luego en abril se observó nuevamente un aumento de población en los tres cultivares estudiados.

Castaño (1996) concluye mediante observaciones de campo, que el minador se ve favorecido por la abundancia de cultivares que presentan diferentes épocas de brotación, por variedades y clones cultivados a diferentes alturas sobre el nivel del mar, por los sistemas de riego y fertilización, que inducen brotaciones periódicas, así como prácticas de poda y cortes de ramas que generan nuevos brotes.

Al relacionar la fluctuación poblacional del minador con la precipitación (figura 6) se observó una relación directa, por tanto, la población aumenta a medida que la precipitación se incrementa y viceversa. Resultados similares se han reportado en zona del Piedemonte y los Llanos Orientales, en periodos de baja precipitación, se reduce la población de insectos.
Durante el estudio no se determinó, la acción de predadores y parasitoides, sin embargo de una muestra de hojas con minas activas, emergió el adulto de un parasitoide identificado como Elasmus sp. (Hymenoptera: Eulophidae) (figura 7), así mismo a nivel de campo se identificaron como predadores importantes de larvas, la avispa Polybia occidentalis (Hymenoptera: Vespidae) (figura 8), considerado un predador importante por su agresividad para destruir larvas dentro de las minas, el Chrysoperla sp. (Neuroptera: Chrysopidae) y dos micro-arañas Aranaeae, una de la familia Salticidae.

Rojas y García (1996) al estudiar el control biológico natural del minador del follaje de los cítricos en el Valle del Cauca, encontraron que la plaga cuenta con una rica y variada fauna benéfica natural, representada principalmente por ectoparasitoides, que pertenecen al orden Hymenoptera y a la familia Eulophidae, pero, no mencionan la especie encontrada en Bahia Concha. Así mismo mencionan una alta depredación por avispas, arañas y crisopas, lo cual concuerda con lo observado en el presente trabajo.

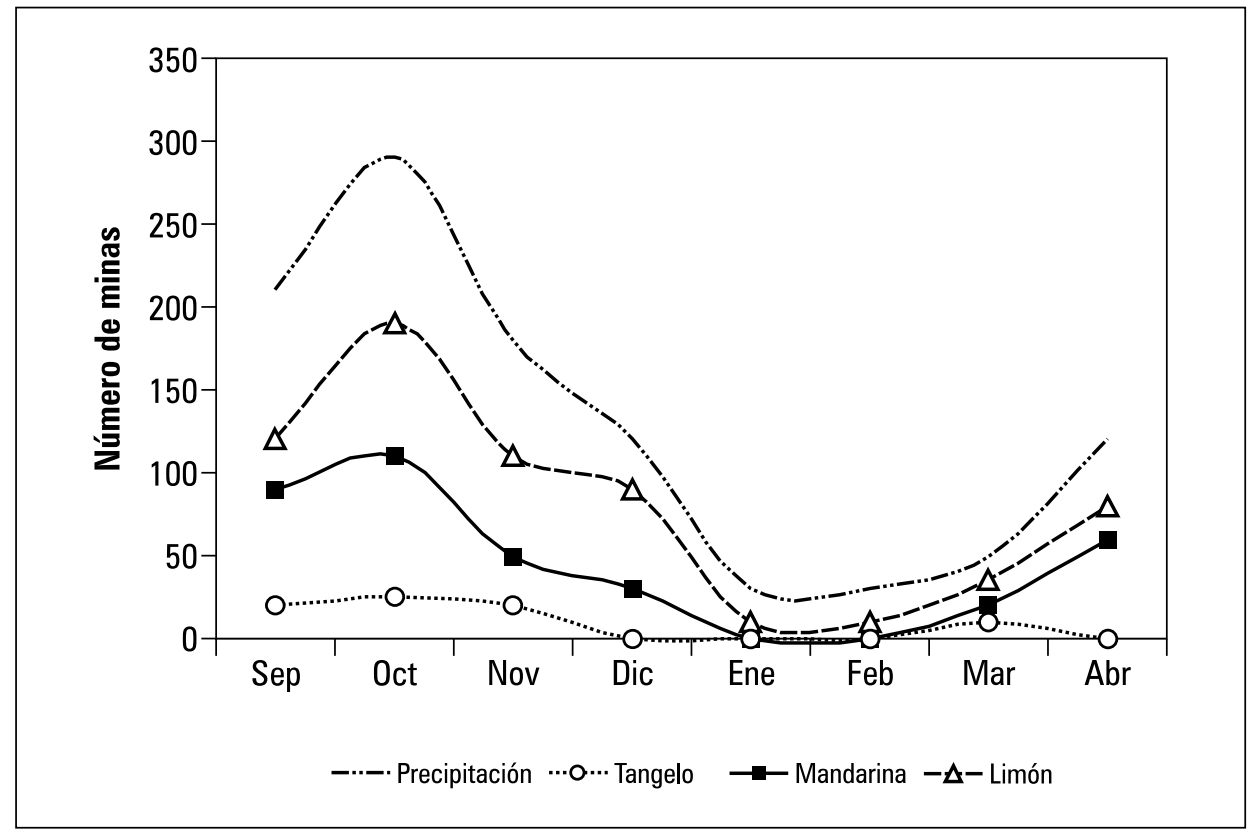

Figura 6. Relación de la población de $P$. citrella en diferentes especies de cítricos en Bahia Concha, municipio de Santa Marta, Magdalena, 2002-2003. 


\section{CONCLUSIONES}

- Phyllocnistis. citrella está ocasionando en Bahia Concha, municipio de Santa Marta, un daño promedio de $35,90 \%$ en el follaje de cítricos, siendo limón el más afectado con un $47 \%$ en el estrato medio de los árboles.

- La población del insecto está directamente correlacionada con la precipitación que favorece la aparición de nuevos rebrotes.
- Observaciones de campo mostraron que la avispa Polybia occidentalis es el predador más importante que tiene el minador en la zona y que el Elasmus sp. fue el único parasitoide emergido de muestras de hojas con minas activas llevadas al laboratorio.

- Por primera vez se hacen estos reportes sobre el $P$. citrella, su daño, infestación, fluctuación poblacional y enemigos naturales en la Costa Atlántica.

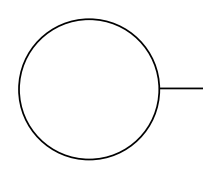

\section{REFERENCIAS BIBLIOGRÁFICAS}

Debach, P. 1968 Control biológico de insectos plagas y malas hierbas. Primera Edición. Compañía Editorial Continental S.A., México. 929 p.

Bustillo, A. 1970 La mosca negra de los cítricos, un problema severo en el Tolima. Agricultura Tropical 26(10), 720-722.

Carrero, J. y S. Planes. 1994 Plagas del campo. Edición número 12. Editorial Mundi-Prensa, Madrid. pp. 359-547.

Castaño, O. 1995. El minador de los cítricos. Boletín Informativo $\mathrm{N}^{\circ} 2$. Asociación Nacional de Productores de Cítricos, Pereira. 85 p.

Castaño, O. 1996. El minador de las hojas de los cítricos (Phyllocnistis citrella Stainton). Memorias XXIII Congreso de la Sociedad Colombiana de Entomología. Cartagena de Indias, julio 17-19, 1996. pp. 9-23.

Cobo, M.G. y A. Tróchez. 1996a. Fluctuación del minador de la hoja de los cítricos Phyllocnistis citrella Stainton (Lepidoptera: Gracillaridae) con relación a su control natural y a la fisiología del cultivo). Memorias XXIII Congreso de la Sociedad Colombiana de Entomología. Cartagena de Indias, julio 17-19, 1996.

Cobo, M.G. y A. Tróchez. 1996b. Ciclo biológico y hospederos del minador de la hoja de los cítricos Phyllocnistis citrella Stainton (Lepidoptera: Gracillaridae) en el Valle del Cauca. Resúmenes XXIII Congreso So- ciedad Colombiana de Entomología. Cartagena de Indias, julio 17-19, 1996. pp. 108.

Espadas, A.L. 1995 El minador de las hojas de los cítricos. Phyllocnistis citrella Stainton. Estrategias para un control eficaz. Phytoma $N^{0} 68,85$ p.

García, G.E. 1995 Metodología para el control del minador de los brotes de los cítricos Phyllocnistis citrella Stainton, Estrategias para un control eficaz. Phytoma $\mathrm{N}^{\circ} 68,85 \mathrm{p}$.

Garrido, A. 1995. El Phyllocnistis citrella Stainton, aspectos biológicos y enemigos naturales encontrados en España. Levante Agrícola, 13-21.

Peña, J.E. y R. Duncan. 1993. Control of citrus leaf miner in South Florida. Proc. Fla. State Hort Soc. 106 p.

Peña, J.E. 1995 Informe de visita realizada a Colombia. Asocítricos, Pereira.

Rojas, A.J. y R.F. García. 1996. Control biológico natural del minador del follaje de los cítricos Phyllocnistis citrella Stainton (Lepidoptera: Gracillaridae). Resúmenes XXIII Congreso Sociedad Colombiana de Entomología. Cartagena de Indias, julio 17-19, 1996. pp. 110.

Suárez, G.H. y P.A. Marín. 2002. Los enemigos naturales de los insectos plagas. Colección Manuales de la Ingeniería Agronómica. Facultad de Ingeniería de Recursos Naturales, Universidad del Magdalena, Santa Marta. Manual Técnico No 1. 20 p. 